with histologically/cytologically confirmed, previously treated, advanced solid tumors with HRRm and/or HRD per Lynparza HRR-HRD assay (Foundation Medicine, Inc., Cambridge, MA, USA), with an ECOG PS of 0-1. Patients will be grouped by biomarker status: subgroup 1: BRCAm; subgroup 2: HRRm without BRCAm; and subgroup 3: HRD positive without HRRm (loss of heterozygosity score $\geq 16$ per Lynparza HRRHRD assay). Patients will receive olaparib $300 \mathrm{mg}$ twice daily + pembrolizumab $200 \mathrm{mg}$ intravenously Q3W (35 cycles) until PD, unacceptable AEs, intercurrent illness, investigator decision, withdrawal of consent, or pregnancy. Tumor imaging assessment by blinded independent central review (BICR) per RECIST v1.1 or Prostate Cancer Working Group (PCWG)modified RECIST v1.1 for prostate cancer will occur Q9W for 12 months, then Q12W until PD, start of new anticancer treatment, withdrawal of consent, pregnancy, or death. AEs will be monitored throughout the study and for 30 days after final dose (90 days for serious AEs). The primary endpoint is ORR (RECIST v1.1 or PCWG-modified RECIST version 1.1 by BICR). Secondary endpoints include duration of response (DOR) and PFS (RECIST v1.1 or PCWG-modified RECIST v1.1 by BICR), OS, and safety. Point estimate and exact Clopper-Pearson CI for ORR, and Kaplan-Meier estimates for DOR, PFS, and OS will be calculated. A total of 89 sites are currently enrolling in 20 countries.

Results N/A

Conclusions N/A

Trial Registration ClinicalTrials. gov identifier, NCT04123366 Ethics Approval An independent institutional review board or ethics committee approved the protocol at each study site, and the trial is being conducted in compliance with Good Clinical Practice guidelines and the Declaration of Helsinki.

http://dx.doi.org/10.1136/jitc-2020-SITC2020.0318

\section{PHASE II TRIAL OF IMMUNOTHERAPY IN PRIMARY GLIOBLASTOMA: ANTIGENS FROM SELF-RENEWING AUTOLOGOUS TUMOR CELLS PRESENTED BY AUTOLOGOUS DENDRITIC CELL VACCINE}

\footnotetext{
${ }^{1}$ Daniela Bota*, ${ }^{3}$ David Piccioni, ${ }^{4}$ Christopher Duma, ${ }^{5}$ Renato LaRocca, ${ }^{6}$ Santosh Kesari, ${ }^{6}$ Jose Carrillo, ${ }^{7}$ Robert O'Donnell, ${ }^{8}$ Robert Aiken, ${ }^{2}$ Frank Hsu, ${ }^{2}$ Xiao-Tang Kong, ${ }^{2}$ Thomas Taylor, ${ }^{9}$ Gabriel Nistor, ${ }^{10}$ Robert Dillman. ${ }^{1}$ Unviersity of California, Irvine, Orange, USA; ${ }^{2}$ UCI, Orange, USA; ${ }^{3}$ UCSD, San Diego, CA, USA; ${ }^{4}$ Hoag Hospital, Newport Beach, CA, USA; ${ }^{5}$ Norton Cancer Center, Louisville, KY, USA; ${ }^{6}$ John Wayne Cancer Institute, Santa Monica, California, USA; ${ }^{7}$ UCDavis, Davis, USA; ${ }^{8}$ Rutgers, New Brunswick, NJ, USA; ${ }^{9}$ Aivita Biomadical, Irvine, CA, USA; ${ }^{10}$ Aivita Biomedical, Irvine, California, USA
}

Background Primary glioblastoma (GBM) is associated with poor survival. Adjunctive vaccines may improve survival by inducing or enhancing anti-GBM immune responses.

Methods A multi-institutional phase II clinical trial was conducted with a primary objective of $75 \%$ survival 15 months after intent-to-treat enrollment. Key eligibility criteria were: (1) primary GBM diagnosis, (2) age $<70$ years at time of tumor resection, (3) successful GBM cell culture, (4) successful monocyte collection by leukapheresis, (5) Karnofsky Performance Status (KPS) > 70 after surgical recovery. Dendritic cells (DC) were differentiated from autologous monocytes, then incubated with autologous tumor antigens (ATA) from the GBM cell line-lysate to produce each patient-specific DC-ATA vaccine. Doses were suspended in $500 \mathrm{mcg}$ granulocyte-macrophage colony-stimulating factor (GM-CSF) at the time of subcutaneous injections at weeks 1, 2, 3, 8, 12, 16, 20 and 24. Patients were enrolled just prior to starting standard concurrent temozolomide (TMZ) and radiation therapy (RT) for the intent-to-treat after recovery from RT/TMZ.

Results Tumors were collected August 2018-January 2020. Cell line success rate was 71/73 (97\%); monocyte collection success rate was 63/65 (97\%), but 10 patients required a second leukapheresis. Patients were enrolled for in-to-treat October 2018-February 2020. The 60 patients included 42 men and 18 women with median age of 59 years (range of 27-70). Racial make-up was 43 White, 10 Hispanic, 2 Black, 1 Asian and 3 Other. KPS was 100 in 4, 90 in 25, 80 in 17 and 70 in 14 (mean 83.2). MGMT methylation was present in 13, absent in 31 , and unknown in 16; IDH mutation was present in 7 , absent in 50, and unknown in 3. 57 patients had received 380 doses with 9 still under treatment at time of abstract submission. 32 had completed all 8 doses; 16 had received fewer than 8 doses when they discontinued treatment. No patient discontinued treatment because of toxicity, but 28 have been hospitalized for 53 treatment-emergent central nervous system-related serious adverse events including seizures (15 episodes), falls and/or increased focal weakness (13 episodes), or severe headaches or visual changes (3 episodes).

Conclusions This patient-specific DC-ATA approach is feasible and may be increasing intratumor inflammation that is associated with on-target efficacy and/or toxicity. An interim survival analysis will be conducted in October 2020, 15 months after the median patient was enrolled; results will be available November 2020 as will immunologic data for 55 patients who received at least two injections.

Trial Registration Clinicaltrials. gov NCT03400917.

Ethics Approval The study was approved by UCI IRB, approval number 2018-4148.

http://dx.doi.org/10.1136/jitc-2020-SITC2020.0319

\section{PHASE IIA STUDY OF ALPHA-DC1 VACCINE AGAINST HER2/HER3, CHEMOKINE MODULATION REGIMEN AND PEMBROLIZUMAB IN PATIENTS WITH ASYMPTOMATIC BRAIN METASTASIS FROM TRIPLE NEGATIVE OR HER2+ BREAST CANCER} ${ }^{1}$ Kristopher Attwood, ${ }^{1}$ Ellis Levine, ${ }^{1}$ Tracey O'Connor, ${ }^{1}$ Amy Early, ${ }^{1}$ Robert Fenstermaker, ${ }^{1}$ Dheerendra Prasad, ${ }^{1}$ Kazuaki Takabe, ${ }^{2}$ Brian Czerniecki, ${ }^{1}$ Pawel Kalinski. ${ }^{1}$ Roswell Park Comprehensive Cancer Center, Buffalo, NY, USA; ${ }^{2}$ Moffitt Cancer Center, Tampa, FL, USA; ${ }^{3}$ Washington University, St. Louis, MO, USA

Background Brain metastases develop in up to $50 \%$ patients with metastatic triple negative breast cancer (TNBC) and HER 2 + BC and are an increasing source of morbidity and mortality. HER3, overexpressed in triple negative and HER2 + brain metastatic breast cancer (BMBC), is a resistance factor to HER2-targeted therapies and a driver of CNS metastasis. Disease progression is associated with loss of anti-HER2/ 3 immunity. We have demonstrated that alphaDC1 loaded with glioma-specific peptides induce intratumoral production of chemokines (CXCL9, CXCL10, CXCL11, CCL5) which attract CXCR3- and CCR5- expressing cytotoxic T-lymphocytes (CTLs) and T-helper 1 (Th1) cells to brain tumors, inducing clinical responses and long-term disease stabilization 
in patients with aggressive recurrent primary brain tumors. Our preclinical data show that Chemokine modulating (CKM) regimen [rintatolimod, interferon (IFN)- $\alpha 2 b$ and COX-2 inhibitor] also selectively attracts effector CTLs and Th1 cells (but not suppressive regulatory T-cells or myeloidderived suppressor cells) into tumors. Importantly, CKM preferentially promotes CTL migration into tumor rather than healthy tissues, providing rationale for its systemic use. We hypothesize that anti-HER2/3 type 1 polarized DC1s in combination with CKM and anti-PD1 will result in improved Th1/CTL response against HER2/3 epitopes, reduce brain recurrence and systemic progression.

Methods This is a phase II single-arm, non-randomized multicenter study (NCT04348747). Eligibility includes patients with triple negative and HER2 + BMBC $\geq 18$ years, ECOG PS $\leq$ 1 , normal marrow and organ function with asymptomatic untreated brain metastases who receive $\alpha \mathrm{DC} 1 \mathrm{q} 2$ weeks $\mathrm{x} 3$, with CKM [200 mg IV rintatolimod, IFN- $\alpha 20$ million units/ $\mathrm{m} 2 \mathrm{IV}$, celecoxib $200 \mathrm{mg}$ oral BID] on days 1-3 with second and third dose of $\alpha \mathrm{DC} 1$, followed by pembrolizumab $200 \mathrm{mg}$ IV. Thereafter, pembrolizumab is given every 3 weeks, along with $\alpha \mathrm{DC} 1$ and CKM every 3 months as booster dose until disease progression, intolerable side effects or withdrawal from study, or up to 24 months. Baseline and 3-week post-CKM treatment peripheral (non-CNS) biopsies are required for six patients. Primary objective is CNS response rate (RR) using RANO-BM criteria. If no CNS response is observed after 12 patients, study will be terminated. If $\geq 1$ response observed, then 9 more patients will be enrolled, for a total of 21 patients. If $\geq 3 \mathrm{CR}$ observed, the proposed therapy will be considered promising for further study. Secondary objectives include non-CNS RR per RECIST v1.1, median CNS, nonCNS and overall progression-free survival, overall survival and safety. Analysis of change in intratumoral biomarkers is an exploratory objective.

Results N/A

Conclusions N/A

Trial Registration NCT04348747

Ethics Approval The study was approved by Roswell Park Comprehensive Cancer Center Institution's Ethics Board, approval number I-19-04120.

http://dx.doi.org/10.1136/jitc-2020-SITC2020.0320

\section{PHASE I CLINICAL TRIAL ASSESSING THE COMBINATION OF SYSTEMIC CHEMOKINE MODULATORY REGIMEN TARGETING TLR3 WITH NEOADJUVANT CHEMOTHERAPY IN TRIPLE NEGATIVE BREAST CANCER}

${ }^{1}$ Shipra Gandhi*, ${ }^{2}$ Mateusz Opyrchal, ${ }^{1}$ Cayla Ford, ${ }^{1}$ Victoria Fitzpatrick, ${ }^{1}$ Melissa Grimm, ${ }^{1}$ Per Basse, ${ }^{1}$ Marie Quinn, ${ }^{1}$ Agnieszka Witkiewicz, ${ }^{1}$ Kristopher Attwood, ${ }^{1}$ Marc Ernstoff, ${ }^{1}$ Tracey O'Connor, ${ }^{1}$ Amy Early, ${ }^{1}$ Ellis Levine, ${ }^{1}$ Pawel Kalinski. ' Roswell Park Comprehensive Cancer Center, Buffalo, NY, USA; ${ }^{2}$ Washington University, St. Louis, MO, USA

Background Neoadjuvant chemotherapy (NAC) with taxanes is the standard of care in triple negative breast cancer (TNBC). Intratumoral prevalence of CD8 + cytotoxic T-lymphocytes (CTLs) is associated with an improvement in relapse-free survival (RFS) and overall survival (OS), while regulatory T-cells (Treg) and myeloid derived suppressor cells (MDSC) are associated with poor survival. Higher ratio of $\mathrm{CTL} /$ Treg is associated with higher probability of obtaining pathological complete response (pCR), a surrogate marker for RFS. Intratumoral production of CCL5, CXCL9, CXCL10 and CXCL11 is critical for local infiltration with CTLs, while CCL22 is responsible for Treg attraction. Previous studies have shown that CXCL9 expression in the pre-treatment breast tissue is associated with a three-fold higher rate of achieving pCR. Our preclinical data show that Chemokine modulating (CKM) regimen, combining rintatolimod (TLR3 agonist), interferon (IFN)- $\alpha 2 b$, and celecoxib (COX-2 inhibitor) increases CTL-attracting, and decreases MDSC-, Tregfavoring chemokines, increasing CTL/Treg ratio in tumor microenvironment, with preferential tumor tissue activation than adjacent healthy tissues. We hypothesize that the combination of CKM with paclitaxel will result in infiltration of TNBC with CTLs, and along with doxorubicin/cyclophosphamide (AC), result in higher pCR, translating into improved RFS and OS.

Methods In this phase I study NCT04081389, eligibility includes age $\geq 18$ years, confirmed resectable TNBC, radiographically measurable disease $\geq 1 \mathrm{~cm}$, ECOG PS $\leq 2$, adequate organ and marrow function. Patients with autoimmune disease, serious mood disorders, invasive carcinoma within 3 years, history of peptic ulcers or hypersensitivity to NSAIDs will be excluded. We plan to treat three patients with early stage TNBC with paclitaxel $80 \mathrm{mg} / \mathrm{m} 2$ IV weekly for 12 weeks, rintatolimod $200 \mathrm{mg}$ IV, celecoxib $200 \mathrm{mg}$ oral twice daily, and accelerated titration of IFN- $\alpha 2 \mathrm{~b}$ at doses 0 , 5, or 10 million units (MU)/m2 [Dose Levels (DL) 1, 2 and 3 respectively] on days 1-3 (no intra-patient dose escalation) in weeks $1-3$. Dose-limiting toxicity (DLT) is defined as grade 3 or higher toxicities within the first 3 weeks. Any DLT will mandate recruitment per the $3+3$ model. If no DLT, three patients will be enrolled at DL 4 at $20 \mathrm{MU} / \mathrm{m} 2$ IFN- $\alpha 2 \mathrm{~b}$. This will be followed by standard dose-dense AC, and then surgery. The primary endpoint is safety and tolerability of combination and to identify the appropriate DL of CKM and paclitaxel for extended efficacy study. The secondary endpoints include investigation of efficacy ( $\mathrm{pCR}$ and breast MRI response), along with RFS and OS. Intratumoral biomarkers will be analyzed in an exploratory manner.

Results N/A

Conclusions N/A

Trial Registration NCT04081389

Ethics Approval The study was approved by Roswell Park Comprehensive Cancer Center Institution's Ethics Board, approval number I-73718.

http://dx.doi.org/10.1136/jitc-2020-SITC2020.0321

\section{EFFICACY AND SAFETY OF GX-17 PLUS PEMBROLIZUMAB FOR HEAVILY PRETREATED PATIENTS WITH METASTATIC TRIPLE NEGATIVE BREAST CANCER: THE PHASE 1B/2 KEYNOTE-899 STUDY}

${ }^{1}$ Joo Sohn*, ${ }^{2}$ Young Hyuk Im. 'Severance Hospital, Seoul, Korea, Republic of; ${ }^{2}$ Samsung Medical Center, Seoul, Korea, Republic of

Background Pembrolizumab monotherapy showed 9.6\% ORR and did not significantly improve $\mathrm{OS}$ as $2 \mathrm{~L}$ or $3 \mathrm{~L}$ treatment for mTNBC compared to standard chemotherapy in phase 3 study (KEYNOTE-119) leading to high unmet needs of a new drug that could enhance the activity of pembrolizumab 